\title{
Un percorso di storia della matematica nella scuola media: la quadratura di figure piane
}

\author{
An experience of History of Mathematics in lower \\ secondary school: the quadrature of plane figures
}

\author{
Vittoria Fontana Bollini e Giovanna Lepori
}

Scuola media - Bellinzona 1 - Svizzera

Sunto / Il presente contributo descrive un percorso didattico di matematica rivolto ad allievi di quarta media ${ }^{1}$. Il progetto ha lo scopo di studiare la possibile quadratura di alcune figure piane, inserendo l'attività nel suo contesto storico, dai matematici greci del V secolo a.C. fino a Ferdinand Lindemann nel 1882. Grazie all'esplorazione e alla collaborazione, gli allievi giungono a realizzare un fascicolo con la quadratura dei principali poligoni da destinare a compagni di altre classi.

Parole chiave: scuola media; quadratura; costruzioni geometriche; situazione - problema; collaborazione tra pari.

\begin{abstract}
This contribution describes a didactic experience in mathematics for ninth grade students. The project aims to study the possible quadrature of some plane figures, framing the activity in its historical context, from Greek mathematicians of the fifth century BC to Ferdinand Lindemann in 1882. Thanks to exploration and collaboration, the students come to realize a booklet with the quadrature of the main polygons meant for schoolmates of other classes.
\end{abstract}

Keywords: lower secondary school; quadrature; geometrical constructions; realistic situation; peer collaboration.

In questo articolo si descrive il percorso sulla quadratura di figure piane realizzato in due classi di corso attitudinale ${ }^{2}$ di quarta media. La prospettiva è quella della storia della matematica: il tema della quadratura rappresenta un punto di partenza ideale per approfondire la nascita e lo sviluppo del concetto di dimostrazione nel pensiero occidentale. La costruzione geometrica eseguita con riga e compasso, la descrizione dei suoi passi per mezzo del linguaggio e la verifica algebrica dell'equiestensione dei poligoni considerati, permettono agli allievi di confrontarsi con diversi registri semiotici e di applicare fondamentali proprietà di geometria sintetica. Infine, la modalità di lavoro proposta per il percorso, sulla scia della pedagogia per progetti, mira ad attivare competenze collaborative e comunicative. Per sviluppare questo percorso ci siamo riferite ad alcune fonti, tra cui Bunt, Jones e Bedient (1987), D'Amore e Sbaragli (2017) e Odifreddi (2011).

1. In Canton Ticino, dopo le scuole elementari è previsto un ciclo di scuola secondaria della durata di quattro anni.

2. Negli ultimi due anni di scuola media ticinese gli allievi sono suddivisi in classi di due livelli in base alle capacità in matematica e in tedesco: il livello attitudinale e il livello base. 


\section{Quadro concettuale}

La valorizzazione della storia della matematica nella programmazione didattica non è certamente una novità e le sue ricadute, in termini di apprendimento, sono riconosciute da tempo. Si tratta in alcuni casi dell'esposizione aneddotica da parte del docente della vita e delle scoperte effettuate dai matematici; in altri casi invece si presentano attività classiche come la conversione da diversi sistemi numerici del passato (ad esempio i geroglifici egizi e i numeri romani) o l'applicazione di procedure che derivano dall'antichità (come nel caso della misurazione dell'altezza della piramide di Cheope e del crivello di Eratostene). In questo percorso si intende piuttosto dare la possibilità agli allievi di confrontarsi con gli aspetti epistemologici di un problema matematico, di immergerli negli ostacoli che questo ha comportato storicamente e di stimolarli, attraverso una situazione coinvolgente, a superarli. A questo proposito riteniamo utile, prima di affrontare le fasi del percorso, fornire alcune indicazioni preliminari sulle radici storiche della quadratura di figure piane e sulle costruzioni geometriche che verranno utilizzate.

\subsection{Conoscenze storiche}

Le civiltà che precedettero o affiancarono quella greca interpretavano i fenomeni naturali sulla base delle loro credenze mitologiche e religiose. Tali civiltà, ad esempio quella egizia o quella babilonese, svilupparono, allo scopo di facilitare il commercio, I'agricoltura ed altri aspetti della vita quotidiana, una matematica di tipo pratico e dogmatica che forniva ricette e metodi senza giustificare le proprie affermazioni. Per gli storici, un tale approccio era certamente collegato alla struttura politico-sociale di quelle civiltà.

È intorno al 600 a.C., con il fiorire della civiltà greca, che tra i filosofi nasce una diversa visione del mondo, basata sulla ragione. La mente umana diventa l'arma più potente per indagare la natura delle cose e il suo strumento privilegiato è la matematica. Con lo sviluppo della democrazia greca, i cittadini non sono più sottomessi all'autorità ma sono liberi di partecipare al dibattito pubblico. Anche i matematici avvertono per la prima volta il bisogno di giustificare i propri risultati. La matematica diviene allora dimostrativa: non si limita più a fornire delle ricette, ma indaga sul problema ben più importante del perché. Se la matematica fin dall'inizio è stata caratterizzata da due filoni principali, I'aritmetica, con il concetto di "molteplicità" e quindi di numero, e la geometria, con quello di spazio, è pur vero che la matematica greca privilegia questo secondo filone, nel tentativo di evitare lo scoglio delle grandezze incommensurabili.

In ambito geometrico, lo sviluppo della dimostrazione è uno degli episodi più significativi della storia della matematica. Agli inizi la dimostrazione si limitava a coincidere con la costruzione geometrica, effettuata con riga non graduata e compasso. In seguito, alla costruzione fu affiancata la trascrizione scritta dei suoi passaggi. Ben presto i geometri greci compresero che la trascrizione permetteva di generalizzare la procedura di giustificazione, passando dal particolare al generale.

Se Talete (624-545 a.C. circa) è considerato il padre della geometria dimostrativa e Pitagora (570-500 a.C. circa) il più importante matematico greco, è a Ippocrate di Chio, vissuto attorno al 440 a.C., che viene attribuita la più antica dimostrazione matematica giunta fino a noi in una forma presumibilmente autentica: la quadra- 
tura della lunula. Noi non possediamo I'opera di Ippocrate, ma un sommario di Simplicio del 530 d.C. che discute gli scritti del 335 a.C. di Eudemo, il quale a sua volta aveva riassunto l'opera di Ippocrate.

L'importanza che il teorema di Ippocrate riveste nella storia della matematica non è legata unicamente alla presunta autenticità della sua dimostrazione. II fatto è che con la quadratura delle lunule di Ippocrate si generò fra i matematici greci l'errata convinzione che fosse possibile quadrare il cerchio. Ma, nonostante gli sforzi profusi, il problema restò irrisolto fino a quando, nel 1882, il matematico tedesco Ferdinand Lindemann riuscì a dimostrare definitivamente che la quadratura del cerchio è un compito impossibile, trasferendo il problema dall'ambito geometrico a quello numerico (Dunham, 1992).

\subsection{Costruzioni con riga non graduata e compasso}

Uno dei problemi che impegnò i matematici del $V$ secolo a.C. fu quindi quello di quadrare una figura piana. Ma cosa si intende per quadratura di una figura piana? La quadratura di una figura piana è la costruzione con riga non graduata e compasso di un quadrato avente area uguale a quella della figura piana considerata.

L'idea di ricondurre l'area di una figura piana qualsiasi a quella di un quadrato fu un problema particolarmente affascinante per il popolo greco. Quadrare qualsiasi figura piana, anche la più complessa, significava realizzare il sogno di un mondo governato dalla ragione e dall'ordine, sostituendo la regolarità all'irregolarità, la simmetria all'asimmetria, la perfezione all'imperfezione, la razionalità all'irrazionalità.

Gli strumenti utilizzati, la riga non graduata e il compasso, erano strumenti essenzialmente poveri, a disposizione di tutti e alla portata delle tecnologie dell'epoca, ma soprattutto erano strumenti prediletti perché con il primo si poteva costruire la figura più perfetta ad una dimensione, la linea retta, e con il secondo quella a due dimensioni, il cerchio. Con tali strumenti, i matematici dell'epoca realizzarono costruzioni geometriche complesse e ingegnose.

Volendo procedere in maniera del tutto filologica, occorrerebbe tener conto del fatto che, nella matematica greca, il compasso veniva inteso come "collassante" o "molle", cioè incapace di mantenere la sua apertura nel momento in cui si cambiava il centro della circonferenza da tracciare: la possibilità di riportare misure con questo strumento era pertanto preclusa (Montagnoli, 2014).

Vediamo come, con questo vincolo, si può ad esempio costruire un segmento $k$ parallelo e congruente al segmento $f$ (Figura 1). 
Figura 1

Costruzione con riga non graduata e compasso

"collassante" o "molle".

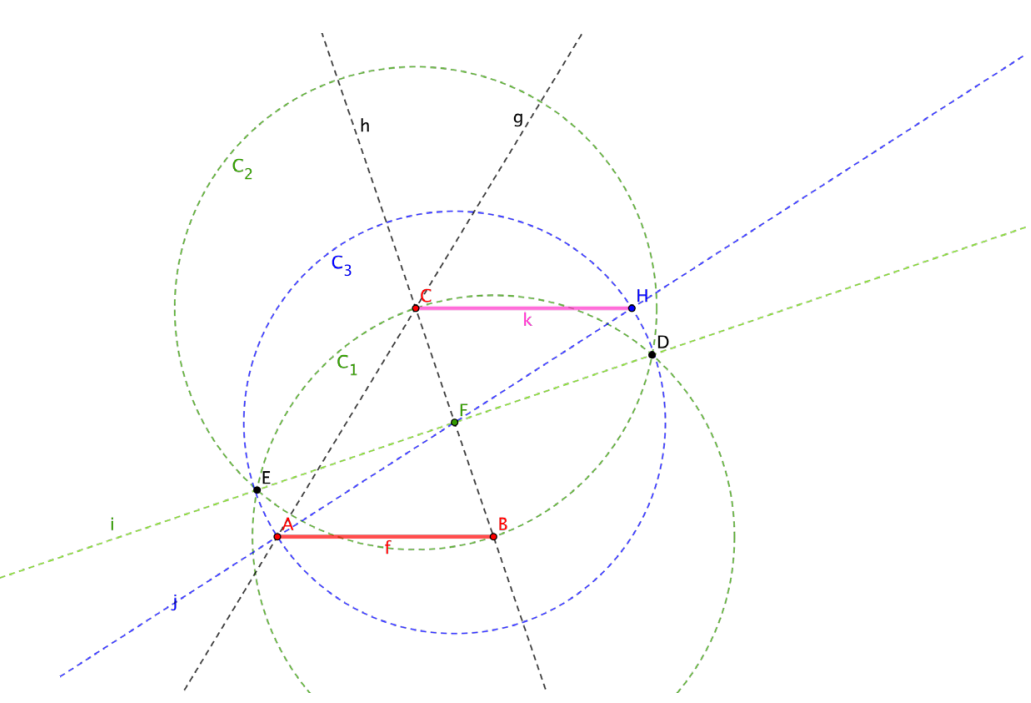

Costruzione del segmento $k$, parallelo al segmento $f$ e ad esso congruente.

1. Sia $f$ il segmento di estremi $A$ e $B$. Sia $C$ un punto non appartenente al segmento $f$.

2. Si tracciano le rette $g$ e $h$ rispettivamente passanti per $A$ e $C$ e per $B$ e $C$.

3. Si tracciano le circonferenze $C_{1}(B ; \overline{B C})$ e $C_{2}(C ; \overline{C B})$; siano $E$ e $D$ i punti di intersezione tra le circonferenze $C_{1}$ e $C_{2}$.

4. Si traccia la retta $i$, passante per i punti $E$ e $D$. Sia $F$ il punto di intersezione delle rette $i$ e $h$. F è punto medio del segmento $B C$.

5. Si traccia la retta $j$ passante per $A$ e $F$ e la circonferenza $C_{3}(F ; \overline{F A})$.

6. Sia $H$ il punto di intersezione della retta $j$ con la circonferenza $C_{3^{\prime}}$ diverso da $A$.

7. Sia $k$ il segmento $\mathrm{CH}$. $k$ è il segmento parallelo a $f$ e ad esso congruente.

Nel percorso realizzato in classe abbiamo deciso di lavorare con un comune compasso e, esplicitando la nostra scelta agli allievi, di utilizzarlo anche per riportare misure. Si può infatti dimostrare che, con il compasso collassante, è possibile tracciare una circonferenza con un raggio pari a un segmento dato. II vantaggio della nostra scelta effettuata in classe è in termini pratici notevole: il numero di passi che caratterizzano le quadrature si riduce notevolmente ma le costruzioni non perdono nulla della loro bellezza.

Vedremo di seguito le costruzioni fondamentali, realizzate con GeoGebra, che a partire dalla quadratura del rettangolo portano a quella delle lunule. Sono in questa sede lasciate al lettore le costruzioni più comuni e frequenti, ad esempio il punto medio di un segmento, la perpendicolare e la parallela ad una retta data passante per un punto.

\subsubsection{La quadratura del rettangolo}

Il primo passo di questo percorso è la quadratura del rettangolo. Il procedimento è descritto nella Proposizione 14 del secondo libro degli Elementi di Euclide (IV secolo a.C. - III secolo a.C.). Nella descrizione del procedimento (Figura 2) si danno per già conosciute la procedura per trovare il punto medio di un segmento e quella per costruire un quadrato dato il lato con riga non graduata e compasso. 


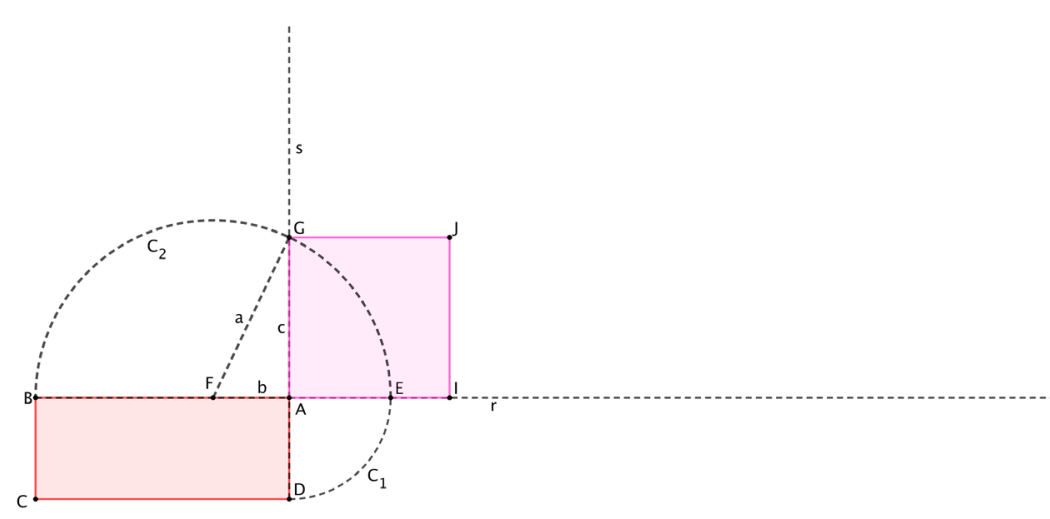

Sia $A B C D$ un rettangolo

1. Si traccia la semiretta $r$ con origine in $B$ passante per $A$.

2. Si traccia la circonferenza $C_{1}(A ; \overline{A D})$ di centro $A$ e raggio $A D$.

3. Sia $E$ il punto di intersezione tra la circonferenza $C_{l}$ e la semiretta $r$.

4. Sia $F$ il punto medio di $B E$.

5. Si traccia la circonferenza $C_{2}(F ; \overline{F E})$ di centro $F$ e raggio $F E$.

6. Si traccia la semiretta $s$ con origine in $D$ passante per $A$.

7. Sia $G$ il punto di intersezione della semiretta $s$ con la circonferenza $C_{2}$

8. Si costruisce il quadrato $A I J G$ di lato $A G$.

Dimostriamo che l'area del rettangolo $A B C D$ è uguale a quella del quadrato $A I J G$.

Sia: $\overline{F G}=a ; \overline{F A}=b ; \overline{G A}=c$. Allora $\overline{B A}=a+b ; \overline{A D}=a-b$.

II triangolo $A G F$ è rettangolo in $A$. Quindi per il teorema di Pitagora: $b^{2}+c^{2}=a^{2}$.

L'area del rettangolo $A B C D: A_{A B C D}=(a+b) \cdot(a-b)$.

L'area del quadrato $A I J G$ : $A_{A I J G}=c^{2}$.

Per il teorema di Pitagora: $A_{A B C D}=(a+b) \cdot(a-b)=a^{2}-b^{2}=c^{2}=A_{A I J G}$.

\subsubsection{La quadratura del triangolo}

Nella costruzione che segue (Figura 3) si danno per conosciute la costruzione con riga non graduata e compasso della retta passante per un punto e perpendicolare a una retta data, la procedura per trovare il punto medio di un segmento e la costruzione con riga non graduata e compasso di un rettangolo dati due segmenti. 
Figura 3

Procedura per la costruzione di un rettangolo equivalente a un triangolo dato.
Figura 4

Suddivisione del poligono in triangoli.
Sia $A B C$ un triangolo.

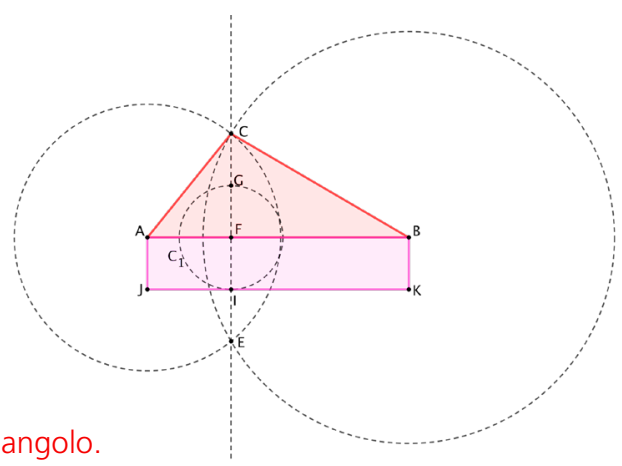

1. Si traccia la retta $r$ passante per $C$ e perpendicolare al segmento $A B$.

2. Sia $F$ l'intersezione tra la retta $r$ e il segmento $A B$.

3. Si trova il punto medio $G$ del segmento $F C$.

4. Sia $I$ il punto di intersezione tra la retta $r$ e la circonferenza $C_{1}(F ; \overline{F G})$ di centro $F$ e raggio $F G$.

5. Si costruisce il rettangolo $A J K B$ di lati $A B$ e $A J$ parallelo e congruente a $\overline{F I}$.

L'area del triangolo $A B C$ è equivalente a quella del rettangolo $A J K B$. La dimostrazione è lasciata al lettore. Una volta costruito il rettangolo equivalente al triangolo di partenza, grazie al procedimento illustrato nel paragrafo precedente si potrà costruire il quadrato equivalente al rettangolo ottenuto. Poiché l'equivalenza gode della proprietà transitiva, il triangolo di partenza sarà equivalente al quadrato così ottenuto.

\subsubsection{La quadratura del poligono}

Consideriamo un poligono qualsiasi, come quello in Figura 4. La procedura per la quadratura di un poligono qualsiasi sfrutta le costruzioni viste nei due paragrafi precedenti, unite alla possibilità di scomporre ogni poligono in triangoli.

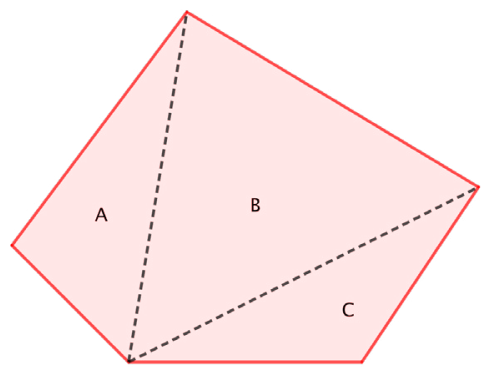

Si tracciano le diagonali del poligono che partono da un singolo vertice, suddividendolo in triangoli di area $A, B, C$. L'area del poligono è quindi $A+B+C$. 
Figura 5

Quadratura dei triangoli che compongono il poligono.

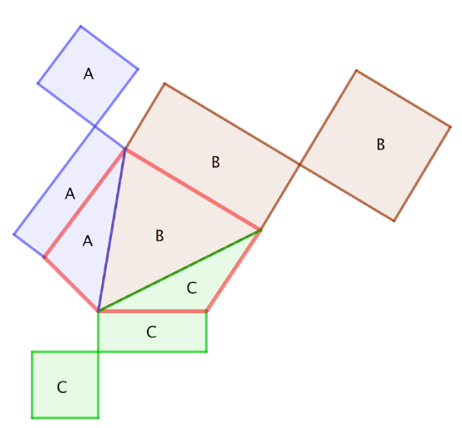

Si quadrano i triangoli di area $A, B, C$ applicando la procedura del paragrafo precedente. I quadrati ottenuti hanno area $A, B, C$.
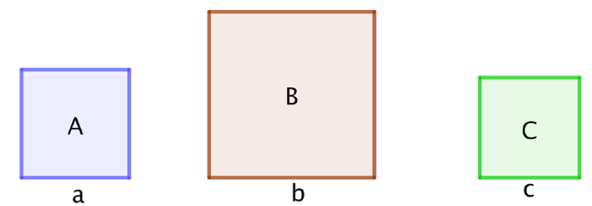

Siano $a, b, c$ i lati dei quadrati di area rispettivamente $A, B, C$.

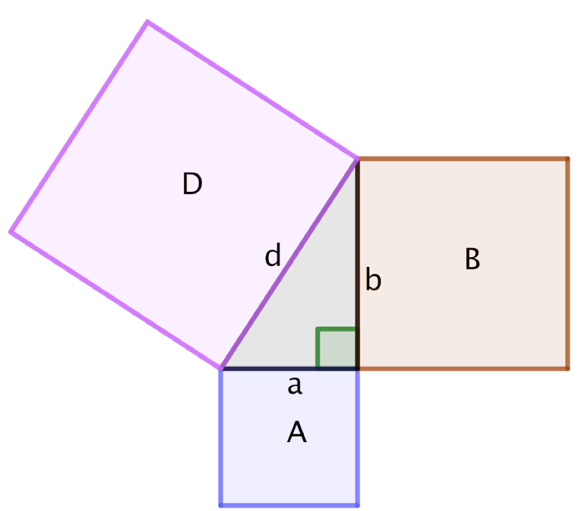

Si costruisce il triangolo rettangolo di cateti $a, b$. Sia $d$ la sua ipotenusa. Si costruisce il quadrato di lato $d$. Per il teorema di Pitagora:

$D=d^{2}=a^{2}+b^{2}=A+B$. 
Figura 8

Costruzione del quadrato $E$ equiesteso al poligono dato.

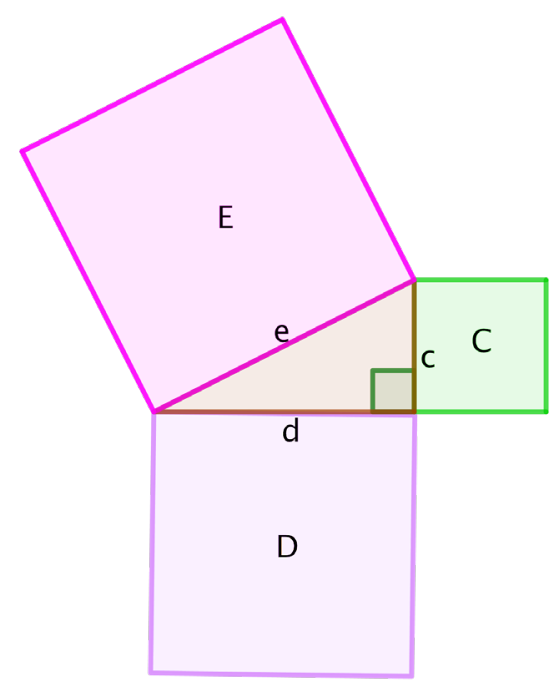

Si costruisce il triangolo rettangolo di cateti $d, c$. Sia $e$ la sua ipotenusa. Si costruisce il quadrato di lato $e$. Per il teorema di Pitagora: $E=e^{2}=d^{2}+c^{2}=D+C$.
Figura 9

Costruzione unica del quadrato $E$ equiesteso al poligono dato.

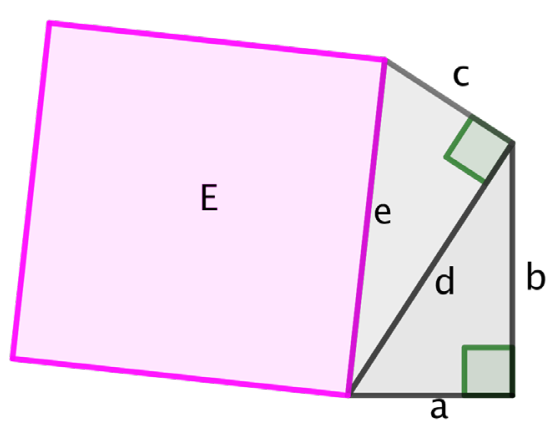

I due passi delle Figure 7 e 8 possono far parte di un'unica costruzione, come si può osservare in questa figura.

Dimostriamo che il quadrato di lato $e$ ha la stessa area del poligono di partenza:

$$
E=e^{2}=d^{2}+c^{2}=a^{2}+b^{2}+c^{2}=A+B+C .
$$

Questa procedura può essere facilmente adattata a un poligono qualsiasi, anche nel caso in cui la figura sia la differenza tra due aree quadrabili, utilizzando ancora il teorema di Pitagora. Nel caso mostrato in Figura 10, infatti, è sufficiente quadrare il triangolo di colore bianco. Una volta fatto ciò, si può utilizzare il teorema di Pitagora per ricavare un quadrato equivalente al poligono di partenza. 

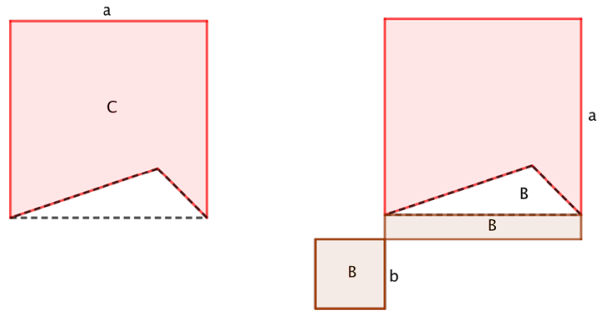

Passo 1

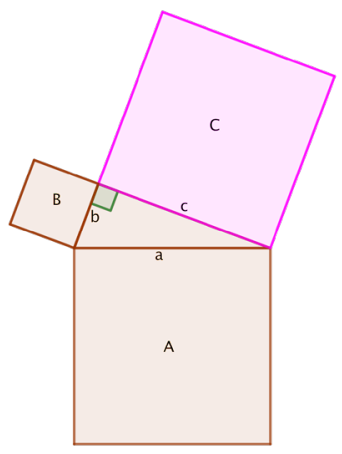

Passo 3

\subsubsection{La quadratura della lunula di Ippocrate}

La lunula è una figura piana delimitata da due archi di circonferenza. Ippocrate non quadrò la lunula in generale, ma alcune particolari lunule, che aveva prima attentamente costruito (Figura 11).

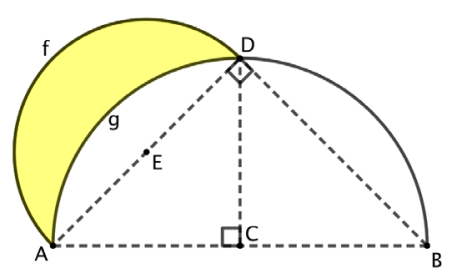

1. Si traccia una semicirconferenza di centro $C$ e diametro $A B$.

2. Si costruisce in essa il triangolo isoscele $A B D$.

3. Sia $E$ il punto medio del segmento $A D$.

4. Si traccia la semicirconferenza $f$ di centro $E$ e diametro $A D$.

5. Si consideri il quarto di circonferenza $g$. La figura racchiusa tra i due archi $f$ e $g$ prende il nome di lunula di Ippocrate.

Il ragionamento di Ippocrate per quadrare la lunula si fonda su tre teoremi:

1. Il teorema di Pitagora.

2. Ogni triangolo inscritto in una semicirconferenza è rettangolo (Talete).

3. Le aree di due cerchi stanno tra loro come i quadrati dei loro diametri.

Ippocrate dimostrò che l'area della particolare lunula (in giallo in Figura 12) è uguale a quella di un triangolo (il triangolo $A C D$, in viola in Figura 12). Avendo già dimostrato la quadrabilità dei triangoli, ne consegue che la lunula è quadrabile. 
Figura 12

Dalla lunula al triangolo.

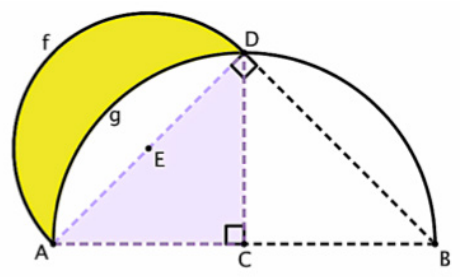

Seguiamo i dettagli della sua dimostrazione.

Per comodità poniamo:

$A_{1}$ : area del semicerchio $A D f \quad A_{3}$ : area del quarto di cerchio $A C D g$

$A_{2}$ : area del semicerchio $A D B \quad A_{4}$ : area del segmento circolare $A D g$

$A_{L}$ : area della lunula $\quad A_{T}$ : area triangolo $A C D$

- Il triangolo $A B D$ è rettangolo e isoscele. Per il Teorema 1:

$(\overline{A B})^{2}=(\overline{A D})^{2}+(\overline{B D})^{2}=2 \cdot(\overline{A D})^{2}$.

- Dato che $A B$ è il diametro del semicerchio $A D B$ e $A D$ è quello del semicerchio $A D f$, per il Teorema 3: $\frac{A_{1}}{A_{2}}=\frac{\overline{A D}^{2}}{A B^{2}}=\frac{\overline{A D}^{2}}{2 \cdot \overline{A D}^{2}}=\frac{1}{2}$.

Ne consegue che:

- L'area del semicerchio $A D f$ è uguale all'area del quarto di cerchio $A C D g$ : $A_{1}=\frac{1}{2} A_{2}=A_{3}$.

- Sottraendo ad $A D f$ e ad $A C D g$ il segmento circolare $A D g$, si ottiene che l'area della lunula è equivalente all'area del triangolo $A C D$ :

$A_{L}=A_{1}-A_{4}=A_{3}-A_{4}=A_{T}$.

Vediamo ora la quadratura di altre lunule, riportate qui sotto, di cui daremo una dimostrazione con gli strumenti algebrici alla portata degli allievi di quarta media.

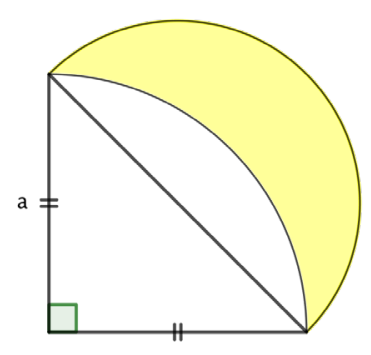

L'area della lunula $\left(A_{L}\right)$ è equivalente a quella del triangolo rettangolo isoscele $\left(A_{T}\right)$ su cui è costruita.

Sia $a$ il cateto del triangolo rettangolo isoscele. L'area della lunula si può ottenere come differenza fra l'area del semicerchio e l'area del segmento circolare. In simboli:

$A_{L}=\frac{1}{2}\left(\frac{a \sqrt{2}}{2}\right)^{2} \pi+\frac{1}{2} a^{2}-\frac{1}{4} a^{2} \pi=\frac{1}{2} a^{2}=A_{T}$ 
Figura 14

Quadratura delle lunule costruite sui cateti di un triangolo rettangolo.

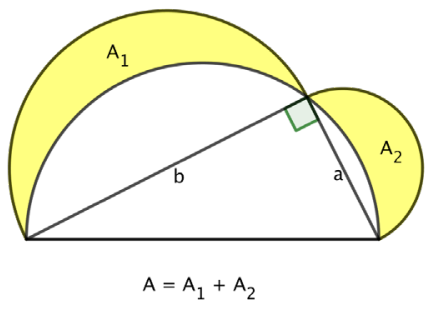

L'area complessiva delle due lunule $(A)$ è uguale all'area del triangolo rettangolo $\left(A_{T}\right)$ su cui sono costruite.

Siano $a, b$ i cateti del triangolo rettangolo.

$$
A=\frac{1}{2} \pi\left(\frac{a}{2}\right)^{2}+\frac{1}{2} \pi\left(\frac{b}{2}\right)^{2}+\frac{a b}{2}-\frac{1}{2} \pi\left(\frac{\sqrt{a^{2}+b^{2}}}{2}\right)^{2}=\frac{a b}{2}=A_{T}
$$

Anche in questi casi, avendo già dimostrato la quadrabilità dei triangoli, ne consegue quella delle lunule.

Ippocrate riuscì, a quanto sembra, a quadrare tre diversi tipi di lunule, mentre nel 1771 il matematico Leonhard Euler trovò altri due tipi di lunule quadrabili.

Alla fine del ventesimo secolo N. G. Tschebatorew e A. W. Dorodnow dimostrarono che queste cinque erano proprio le uniche lunule quadrabili.

\subsubsection{Tentativi di quadrare il cerchio}

Come abbiamo già detto, la quadratura delle lunule di Ippocrate riaccese la speranza, nei matematici, di riuscire a quadrare il cerchio. Molti furono i tentativi che si susseguirono nei secoli. Ne vediamo uno (Figura 15) che Alessandro di Afrodisia (210 circa) attribuì allo stesso Ippocrate.
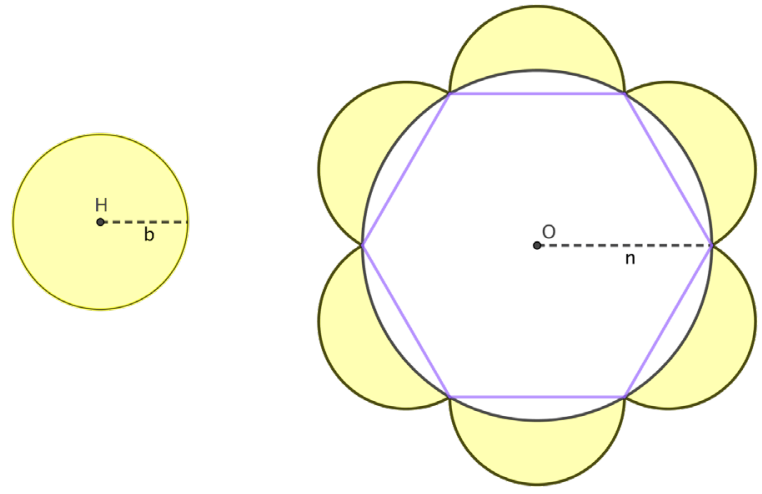

1. Si costruiscono le circonferenze $C_{1}(H ; b)$ e $C_{2}(O ; n)$ con $n=2 b$.

2. Si costruisce l'esagono $E$ inscritto in $C_{2}$.

3. Si costruiscono sei semicirconferenze i cui diametri sono i lati dell'esagono.

Si consideri la regione in giallo formata dal cerchio $C_{1}$ e dalle sei lunule, delimitate dalle sei semicirconferenze e da $C_{2}$. 
Dimostriamo che l'area della regione in giallo è equivalente a quella dell'esagono.

Per comodità poniamo:

$A_{R}$ : area della regione gialla $\quad A_{E}$ : area dell'esagono

$A_{L}$ : somma delle aree delle sei lunule $A_{I}$ : area di $C_{1}$

$A_{2}$ : area di $C_{2} \quad A_{S}$ : area di un semicerchio di diametro $n$

- La figura a destra può essere vista come la composizione del cerchio $C_{2}$ più sei lunule oppure come l'esagono $E$ più i sei semicerchi costruiti sui suoi lati. Da ciò deriva la seguente uguaglianza: $A_{E}+6 \cdot A_{s}=A_{2}+A_{L}$.

- Il raggio del cerchio $C_{2}$ è il doppio di quello di $C_{1}$. Quindi: $A_{2}=4 \cdot A_{1}$.

- Le semicirconferenze hanno lo stesso raggio di $C_{l}$. Quindi: $A_{l}=2 \cdot A_{s}$.

Ne consegue che: $\left\{\begin{array}{l}A_{E}+6 \cdot A_{s}=A_{E}+3 \cdot A_{1} \\ A_{2}+A_{L}=4 \cdot A_{1}+A_{L}\end{array} \quad\right.$ quindi: $A_{E}=A_{1}+A_{L}=A_{R}$.

Conseguenza di questo risultato è che l'area della circonferenza $C_{l}$ è data dalla differenza tra l'area dell'esagono e quella delle lunule.

Secondo Alessandro di Afrodisia, Ippocrate avrebbe ricavato da questo risultato la quadrabilità del cerchio, data la quadrabilità dell'esagono e delle lunule. II vizio del ragionamento sta nel fatto che la quadrabilità delle lunule costruite sull'esagono non è dimostrata. In verità tali lunule non sono quadrabili. Secondo la maggior parte degli studiosi, è improbabile che Ippocrate sia incappato in un errore tanto grossolano.

\subsubsection{La soluzione al problema della quadratura del cerchio}

Come abbiamo già anticipato, la parola fine all'annoso problema della quadratura del cerchio è stata messa dal matematico tedesco Ferdinand Lindemann che nel 1882 dimostrò che il cerchio non è quadrabile, trasferendo il problema dall'ambito geometrico a quello numerico.

Vediamo a grandi linee la sua dimostrazione.

Figura 16

Numeri costruibili

con riga non graduata

e compasso.

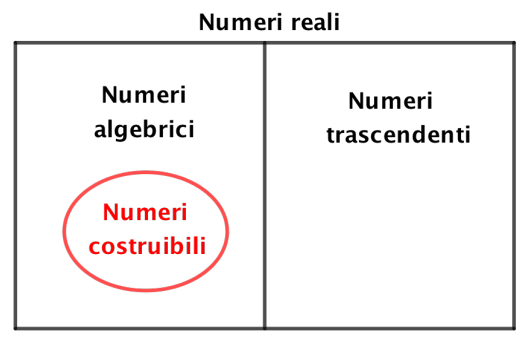

- L'insieme dei numeri reali si suddivide in due insiemi disgiunti: quello dei numeri algebrici, soluzione di un'equazione polinomiale a coefficienti interi, e quello dei numeri trascendenti. L'insieme dei numeri costruibili, con riga non graduata e compasso, è un sottoinsieme dell'insieme dei numeri algebrici (Figura 16). 
- Si consideri il cerchio di raggio 1 e quindi di area $\pi$. Per disegnare un quadrato con pari area occorrerà disegnare il suo lato, di misura $\sqrt{\pi}$.

- Lindemann dimostrò che $\pi$ e $\sqrt{\pi}$ sono numeri trascendenti e quindi non costruibili.

\section{Le fasi del percorso}

Dopo aver ripercorso gli elementi storici e concettuali della quadratura delle figure piane, possiamo addentrarci nella sua trasposizione didattica. In questo articolo verrà presentato l'intero percorso, realizzato in 15 ore lezione, ma è possibile ridurne la durata apportando le modifiche che il docente riterrà necessarie: ad esempio guidando maggiormente l'attività o tralasciando la realizzazione del fascicolo. II percorso è stato realizzato in due corsi paralleli di quarta attitudinale; ciò ha permesso di lavorare, in alcuni momenti a classi unificate e in modalità team teaching: benché per chi scrive rappresenti un valore aggiunto, questa modalità di lavoro non è una condizione necessaria alla realizzazione del percorso.

\subsection{Introduzione}

\subsubsection{La valenza culturale della quadratura}

II percorso sulla quadratura delle figure piane prende avvio da un'analisi di tipo semantico: propone cioè agli allievi, sulla base di testi autentici presenti nel web, un brain storming iniziale sul significato dell'espressione idiomatica "quadrare il cerchio" (Allegato 1).

Con questa riflessione gli allievi hanno la possibilità di avvicinarsi alla valenza culturale della matematica e all'utilizzo dei suoi termini, che, entrando a far parte del linguaggio comune, possono assumere un senso figurato. Gli estratti si riferiscono al mondo del cinema, del calcio e della moda, temi che stimolano la partecipazione degli allievi in quanto vicini ai loro interessi. Può essere sicuramente utile affrontare questa introduzione in collaborazione con il docente di italiano, per approfondire, ad esempio, I'uso delle figure retoriche o del linguaggio settoriale.

In questa prima fase è importante che il docente eviti di prendere posizione sulla possibilità di quadrare il cerchio con riga e compasso, così da mantenere viva la curiosità degli allievi. Nelle nostre classi, ad esempio, era emersa l'ipotesi che l'espressione significasse «riuscire a fare una cosa molto difficile».

Dopo aver sottoposto ad interpretazione l'espressione idiomatica nella sua globalità, si affronta più in dettaglio il significato del verbo "quadrare" dal punto di vista matematico: anche in questo caso, il docente, in forma dialogata, chiede alla classe di formulare delle ipotesi. Gli allievi, spesso, utilizzano il concetto di uguaglianza tra figure geometriche senza essere pienamente consapevoli della grandezza alla quale fanno riferimento. Domande del docente come:

- Cosa intendi per "uguali"? Nella forma?

- Nella lunghezza dei perimetri? Nella misura dell'area della superficie?

- Puoi venire alla lavagna a disegnare due figure che ritieni uguali? Sono tutti d'accordo?

aiutano i discenti a esplicitare la relazione di uguaglianza tra due figure piane. Grazie alla guida e ai feedback del docente, gli allievi giungono a comprendere il significato 
della quadratura in senso geometrico.

Gli studenti affrontano in seguito, a coppie, la lettura della scheda (Allegato 2) nella quale si sottolinea il senso e il valore di questa costruzione nella civiltà dell'antica Grecia e se ne istituzionalizza il significato.

\subsubsection{La quadratura del rettangolo}

Un primo esempio di quadratura con riga non graduata e compasso permette di verificare le conoscenze pregresse necessarie per affrontare il percorso, quali il confronto della misura della superficie di due poligoni e l'utilizzo corretto di riga e compasso per costruzioni di base come riportare segmenti, determinare punti medi e costruire quadrati (Allegato 3). Alla classe, suddivisa in gruppi, viene mostrata l'immagine della quadratura del rettangolo. Agli allievi è richiesto inizialmente di dimostrare l'equiestensione del rettangolo e del quadrato dati per mezzo di un'uguaglianza algebrica (domanda a), e successivamente di riprodurre la costruzione a partire da un nuovo rettangolo (domanda b), elencandone i passi risolutivi (domanda c).

In generale le prime due consegne vengono affrontate da allievi di quarta media senza troppe difficoltà: la dimostrazione algebrica presuppone infatti la conoscenza del teorema di Pitagora e di semplici tecniche di calcolo (vedi par. 2.2.1).

Più ostica risulta invece la terza richiesta della scheda, quella cioè di elencare i passi della costruzione, in quanto gli allievi potrebbero faticare a esplicitare il processo risolutivo seguito e a utilizzare un linguaggio specifico per descrivere procedure di tipo geometrico. Sarà quindi fondamentale, nello svolgimento di tutto il percorso, prestare particolare attenzione allo sviluppo di una terminologia adeguata, mostrando agli allievi con esempi concreti i pericoli di un linguaggio impreciso e la conseguente necessità di utilizzare termini chiari e condivisi. Per dare un esempio concreto delle difficoltà incontrate nella stesura dei passi della quadratura del rettangolo nelle nostre classi, riportiamo la versione redatta da un gruppo di allievi (Figura 17).

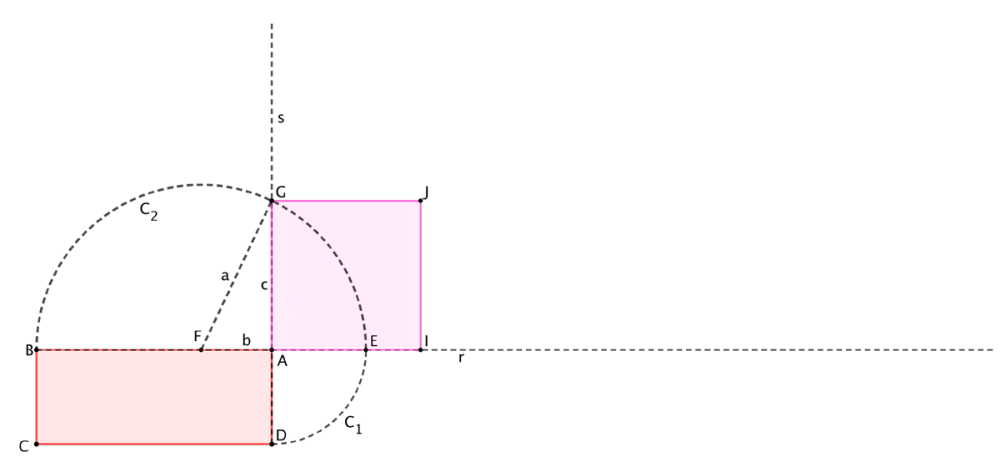

1. Apriamo il compasso su $A D$ e spostiamo il punto $D$ lungo la retta $A B$.

2. Poi troviamo il punto centrale $F$ della retta $A B$ e tracciamo un semicerchio.

3. Prolunghiamo la retta $A D$ fino a raggiungere il semicerchio.

4. Riportiamo con il compasso l'apertura $A G$ spostando la punta sulla retta $A B$, lasciando l'altra punta su $A E$, creando così il punto $I$. 
Attraverso la messa in comune del processo risolutivo adottato e la riflessione sul linguaggio geometrico, verrà costruita una versione finale e il più possibile rigorosa della costruzione, poi riportata su una scheda e consegnata ad ogni allievo all'inizio della lezione successiva (Allegato 4).

Tra gli scopi di questa attività vi è quindi anche quello di lavorare sulla terminologia, affinché possa servire da riferimento per il lavoro successivo: come abbiamo visto in precedenza, la quadratura del rettangolo rappresenta infatti un passaggio chiave per dimostrare equiestensioni di molte altre figure piane.

Questa fase del percorso si conclude con una riflessione del docente di tipo epistemologico sul concetto di dimostrazione (Allegato 5). È infatti importante chiarire agli allievi che la dimostrazione algebrica, come quella da loro utilizzata nella quadratura del rettangolo, ha una storia piuttosto recente nello sviluppo del pensiero scientifico occidentale ed è la conclusione di un lungo percorso iniziato proprio nella Grecia antica (vedi par. 2.1). Anche in questo caso è possibile collaborare con il docente di storia che, ad esempio tramite l'analisi dell'affresco La scuola di Atene di Raffaello, può ripercorrere i tratti salienti della nascita del pensiero razionale e della democrazia ateniese.

\subsection{Situazione - problema}

La situazione - problema, proposta verbalmente alla classe, è la seguente:

Il compito della vostra classe è quello di elaborare un fascicolo, destinato ad allievi di quarta media, con la raccolta delle quadrature delle principali figure piane. Per preparare le bozze del fascicolo avete a disposizione 8 ore lezione, il computer e, naturalmente, la consulenza del docente.

La consegna è volutamente molto aperta e richiama l'idea del lavoro per progetti: al quarto anno di scuola media, gli allievi sentono la forte esigenza di poter organizzare in modo autonomo il proprio apprendimento e scegliere liberamente come affrontare un determinato compito. Questa situazione problema fa inoltre leva sul forte sentimento dell'appartenenza al gruppo, spingendo la classe a cercare le forme migliori di collaborazione per raggiungere l'obiettivo richiesto. Molte sono senz'altro le vie percorribili per un'efficace modalità di lavoro e anche nei nostri due corsi abbiamo potuto osservare due scelte differenti: nella prima classe è stata nominata un'allieva "responsabile" che assegnava ai compagni le figure da quadrare e ne coordinava il lavoro, nella seconda invece si è istaurata una sorta di leadership condivisa, con discussioni e decisioni collettive. In entrambe le classi, le quadrature sono state distribuite in base al numero di lati e affrontate in piccoli gruppi. Gli allievi hanno intuito velocemente che la via maestra per quadrare le diverse figure piane è quella di trasformarle in rettangoli con la stessa area: la buona conoscenza delle proprietà di triangoli, quadrilateri particolari e poligoni regolari ha permesso loro di eseguire la costruzione senza incontrare grandi difficoltà (Figura 18). 


\section{Figura 18}

Quadratura del triangolo isoscele, del trapezio e del pentagono regolare.
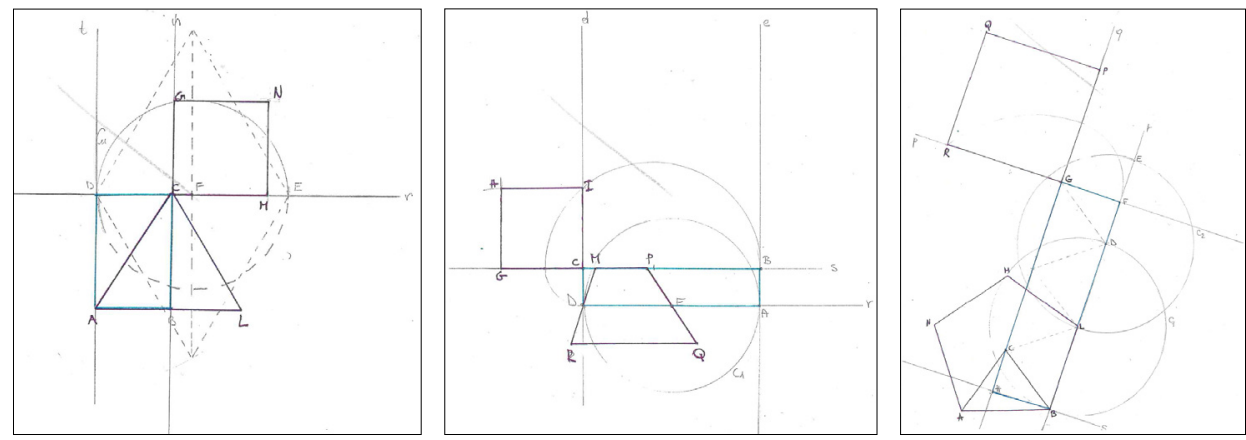

Uno dei nodi centrali dell'attività è stato quello di applicare il teorema di Pitagora per la quadratura dei poligoni generici, confrontando di volta in volta la superficie di tre quadrati (Figura 19). In una classe è stato sufficiente sollecitare una discussione collettiva per attivare questa risorsa, nell'altra è stato necessario un aiuto maggiore da parte della docente.

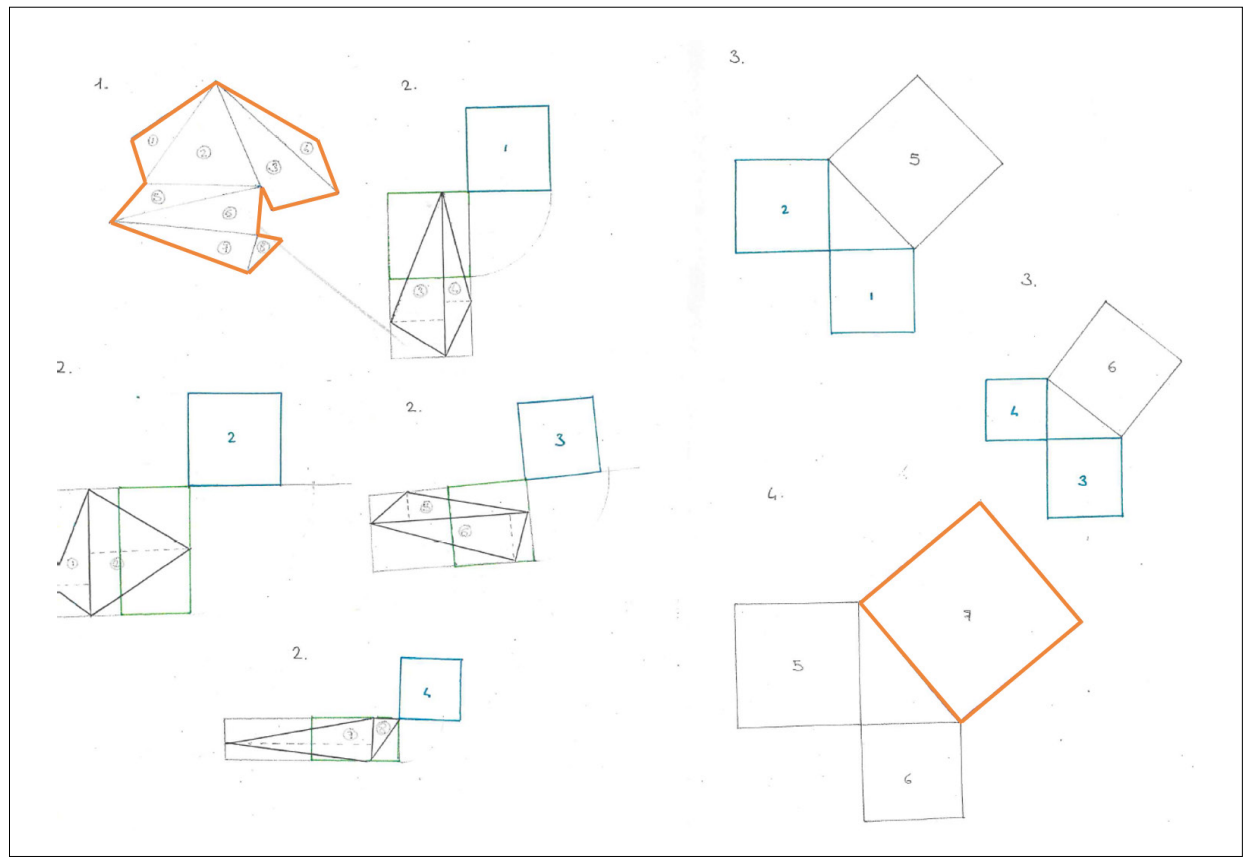

Due sono gli aspetti delicati in questa fase del percorso: da un lato la precisione nel disegno e, come già ribadito, la descrizione dei passi per la costruzione. Come detto in precedenza, I'utilizzo di un linguaggio chiaro e preciso nelle costruzioni geometriche, rappresenta una grande sfida per l'allievo di scuola media, che va quindi accompagnato da vicino. Nel nostro caso è risultato opportuno ritirare il materiale prodotto dai vari gruppi alla fine di ogni lezione e annotare i feedback da fornire all'inizio della lezione successiva: correzioni, riflessioni e spunti che possono venire discussi con tutta la classe o in modo mirato nei diversi gruppi. In questo ultimo caso, per noi è stato utile preparare delle liste di controllo da proporre agli allievi tramite la lavagna luminosa e stimolarli così ad accordarsi su alcune scelte di tipo editoriale o sulla formulazione dei passi che venivano utilizzati con maggior frequenza (Allegato 6).

Gli allievi hanno deciso di redigere i passi delle loro costruzioni con il computer; 
questa scelta, se da un lato ha permesso di migliorare il fascicolo in termini estetici e di chiarezza, dall'altro ha però comportato un necessario approfondimento del tool Equation a disposizione del programma Word. Naturalmente, maggiori saranno la precisione e la completezza delle costruzioni richieste dal docente, maggiore sarà il tempo che gli allievi dovranno dedicare alla preparazione del fascicolo. Anche il grado di pilotaggio da parte del docente influisce notevolmente sulla durata della situazione - problema: la durata del percorso può variare notevolmente se si fornisce agli allievi una rappresentazione iniziale delle figure da quadrare come è stato fatto nel caso del rettangolo.

\subsection{Approfondimento}

Gli allievi, dopo aver affrontato la quadratura delle figure poligonali, si sono interrogati spontaneamente su quella delle figure curvilinee, dalla quale il percorso ha avuto origine. Abbiamo quindi proposto una riflessione a gruppi sulle lunule di Ippocrate, dopo aver introdotto la figura del matematico greco (Allegato 7). La dimostrazione algebrica dell'equivalenza tra l'area delle lunule e quella del triangolo rettangolo su cui sono costruite, rende la costruzione di questa quadratura di semplice esecuzione e si riduce di fatto a quella del triangolo (vedi par. 2.2.4).

È stato interessante notare come i gruppi che avevano lavorato sulla quadratura dei triangoli, si siano in questa occasione messi a disposizione dei loro compagni spontaneamente in qualità di tutor, come nel modello dell'apprendimento cooperativo Jigsaw ${ }^{3}$

Al termine di questa attività, il docente informa la classe dei molti tentativi realizzati nel corso dei secoli per quadrare il cerchio con riga non graduata e compasso e della dimostrazione della sua impossibilità individuata nel 1882 (vedi par. 2.2.6). Diversi allievi delle nostre classi hanno reagito con stupore e un pizzico di delusione a questo finale inatteso della storia della quadratura. Probabilmente, a causa delle aspettative implicite che caratterizzano il contratto didattico, alcuni allievi ritengono che ogni problema posto dal docente porti sempre a una soluzione e sono quindi disorientati dalla non quadrabilità del cerchio per mezzo di strumenti geometrici. Nel corso di una lezione riassuntiva, il docente riprende in forma dialogata gli aspetti disciplinari più rilevanti emersi durante il lavoro: dalle proprietà delle figure piane, necessarie per costruire il rettangolo equiesteso al poligono di partenza, all'applicazione del teorema di Pitagora nel caso di poligoni generici.

\subsection{Realizzazione del fascicolo}

A questo punto del percorso agli allievi della classe non rimane che valutare la veste grafica del fascicolo e realizzarlo. Nel nostro caso gli allievi hanno scelto la copertina, il formato, l'impaginazione e la struttura, decidendo di aggiungere un'appendice con le costruzioni di base. Noi docenti, per una questione di tempi, ci siamo assunte I'incarico di trasformare i loro disegni cartacei in formato pdf, di abbinarli ai passi delle costruzioni già redatti dagli allievi in Word e di stampare i fascicoli così assemblati (Figura 20 e 21). In questa fase è naturalmente possibile, e sicuramente fruttuosa, la collaborazione con la docente di educazione visiva o di arti plastiche.

3. II Jigsaw è una tecnica utilizzata nell'apprendimento cooperativo, ideata negli anni '70. L'idea di base è che ad ogni allievo venga assegnato un compito che è essenziale al gruppo, senza il quale lo stesso non può riuscire a completare un compito o risolvere una situazione problematica. 
Figura 20

Quadratura del pentagono generico e passi della costruzione.
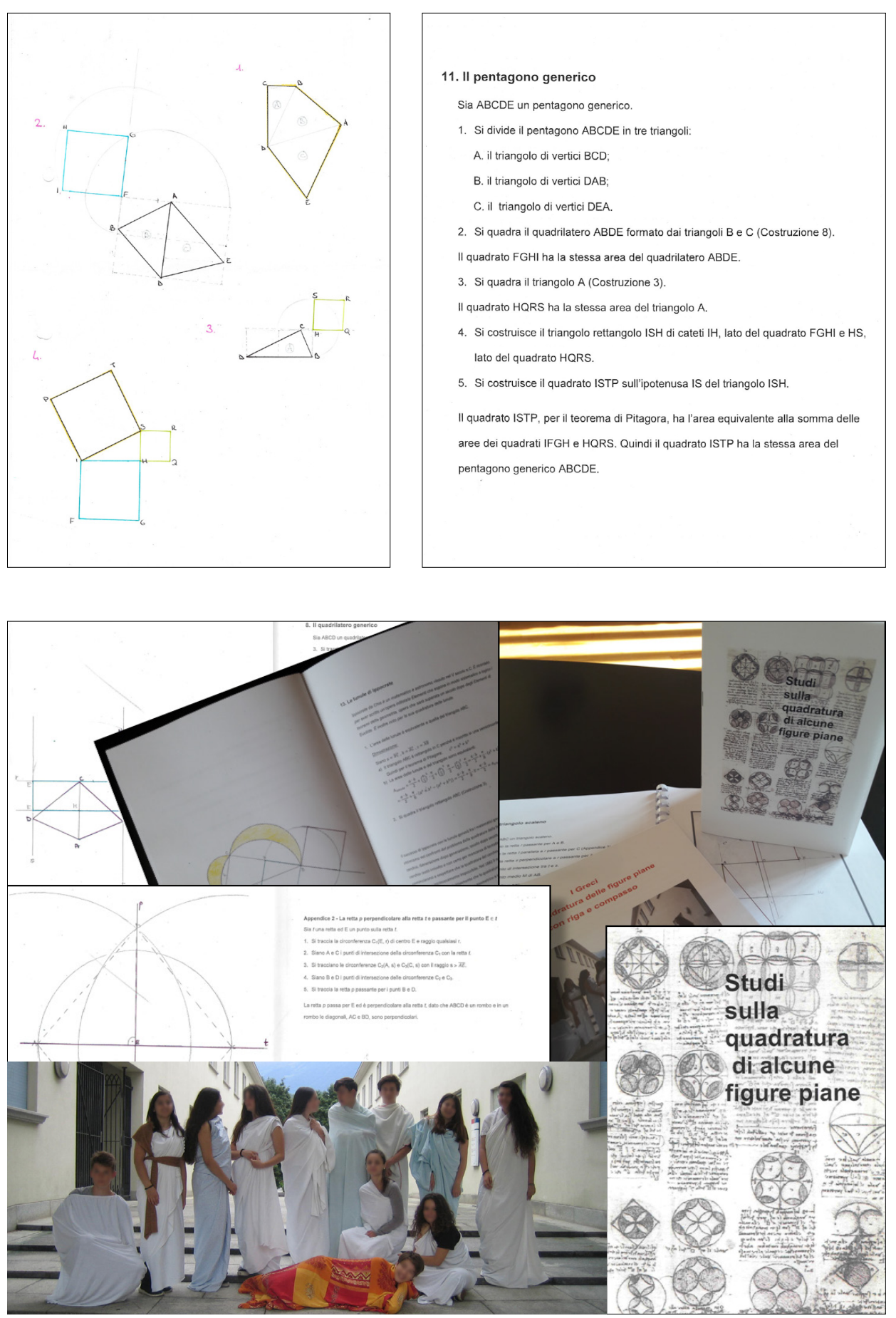

\section{Conclusioni}

Per concludere, desideriamo sottolineare brevemente due aspetti che, nella progettazione di questo percorso, sono stati per noi di grande importanza: I'utilizzo della storia della matematica in funzione dell'apprendimento e la collaborazione tra docenti. La storia della matematica rappresenta una miniera d'oro per costruire una conoscenza della matematica a tutto tondo, in grado di motivare gli allievi attraverso i 
collegamenti interdisciplinari, di accrescere il senso dell'apprendimento e di favorire I'acquisizione di risorse e processi cognitivi. Nel primo biennio di scuola media, il programma di storia tocca una moltitudine di aspetti contigui a quello di matematica: pensiamo, solo per fare alcuni esempi, al calendario delle antiche popolazioni, a trafori e rosoni delle cattedrali gotiche, all'importanza per l'Occidente dell'alto Medioevo delle conoscenze matematiche acquisite dagli Arabi. Lo stesso percorso descritto in questo articolo può essere utilizzato, se opportunamente sfrondato delle sue parti più complesse, anche per avvicinare gli allievi di prima media alla civiltà greca attraverso le costruzioni con riga e compasso e alle proprietà delle figure piane che già conoscono. Ma anche nel secondo biennio le occasioni per lavorare a stretto contatto con la storia non mancano e attività sul teorema di Pitagora o sulla probabilità possono sicuramente trovare un posto nella programmazione annuale della materia. Sulla base della nostra esperienza possiamo ipotizzare che affrontare un argomento di storia della matematica può aumentare la motivazione e la curiosità anche in quegli allievi che sostengono di avere con la matematica, per motivi diversi, un rapporto difficile; per gli allievi più motivati invece esso può rappresentare un forte stimolo a creare nuovi collegamenti tra le diverse branche del sapere. Un percorso come quello della quadratura delle figure piane offre infine anche la possibilità di una differenziazione all'interno della classe, sia nella scelta della figura da quadrare sia nel ruolo da assumere all'interno del proprio gruppo, in modo che ogni allievo, a partire dalle risorse di cui dispone, possa compiere passi avanti nell'apprendimento. La collaborazione ha caratterizzato tutte le fasi della quadratura delle figure piane: il percorso è stato infatti preparato a quattro mani, abbiamo spesso unito le due classi per permettere il co-insegnamento e assieme abbiamo anche affrontato in itinere la preparazione di feedback per i nostri rispettivi allievi. In esperienze analoghe abbiamo inoltre lavorato a stretto contatto con i docenti di storia e realizzato i percorsi in team di più colleghi di matematica. Siamo convinte che la collaborazione tra docenti rappresenti una grande opportunità per creare un forte senso di appartenenza alla professione, per migliorare i propri punti deboli e capitalizzare quelli forti, per sperimentare nuove forme di insegnamento e verificarne l'effetto sugli studenti. In questi anni di team teaching (Fontana Bollini \& Lepori, 2009) abbiamo potuto osservarne anche gli effetti positivi sugli allievi: le differenze tra i nostri stili di insegnamento aumentano la possibilità di stabilire una comunicazione efficace, la presenza di un secondo docente consente una migliore osservazione di quello che succede in aula e offre quindi la possibilità di effettuare interventi più efficaci e il confronto sulle competenze acquisite dagli allievi ne accresce I'affidabilità. In ultimo, siamo convinte che la collaborazione tra docenti abbia una ricaduta diretta anche sul comportamento degli allievi, stimolandoli ad affrontare i momenti di apprendimento cooperativo tra pari con maggior fiducia e disponibilità.

\section{Bibliografia}

Bunt, L., Jones, P. S., \& Bedient, J.D. (1987). Le radici storiche delle matematiche elementari. Bologna: Zanichelli.

D’Amore, B., \& Sbaragli, S. (2017). La matematica e la sua storia. I. Dalle origini al miracolo greco. Bari: Edizioni Dedalo. 
Un percorso di storia della matematica nella scuola media: la quadratura di figure piane /

Vittoria Fontana Bollini e Giovanna Lepori

Dunham, W. (1992). Viaggio attraverso il genio, I grandi teoremi della matematica. Bologna: Zanichelli.

Fontana Bollini, V., \& Lepori, G. (2009). Il team teaching. Bellinzona: CERDD. Disponibile in https://www4.ti.ch/fileadmin/DECS/DS/CERDD/ScuolaLab/Riforma3/Giornate/Bellinzo

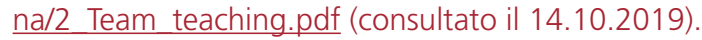

Montagnoli, L. (2014). Appunti di Geometria Elementare. Cattolica: EDUCatt Università Cattolica.

Odifreddi, P. (2011). C'è spazio per tutti. Il grande racconto della geometria. Milano: Mondadori.

Autori/Vittoria Fontana Bollini e Giovanna Lepori

Scuola media, Bellinzona 1 - Svizzera

vittoria.fontana@edu.ti.ch, giovanna.lepori@edu.ti.ch 